\title{
Provision of Two-area Automatic Generation Control by Demand-side Electric Vehicle Battery Swapping Stations
}

\author{
Pingping Xie*, Dongyuan Shi* and Yinhong $\mathrm{Li}^{\dagger}$
}

\begin{abstract}
Application of demand-side resources to automatic generation control (AGC) has a great significance for improving the dynamic control performance of power system frequency regulation. This paper investigates the possibility of providing regulation services by demand-side energy storage in electric vehicle battery swapping stations (BSS). An interaction framework, namely station-to-grid $(\mathrm{S} 2 \mathrm{G})$, is presented to integrate BSS energy storage into power grid for giving benefits to frequency regulation. The BSS can be regarded as a lumped battery energy storage station through S2G framework. A supplementary AGC method using demand-side BSS energy storage is developed considering the vehicle user demand of battery swapping. The effects to the AGC performance are evaluated through simulations by using a two-area interconnected power grid model with step and random load disturbance. The results show that the demand-side BSS can significantly suppress the frequency deviation and tie-line power fluctuations.
\end{abstract}

Keywords: Automatic generation control, Battery swapping station, Demand-side response, Electric vehicle, Energy storage

\section{Introduction}

Automatic generation control is an essential mechanism in electric power system which regulates the output of generation units in response to the load fluctuations in order to maintain the system frequency and tie-line power at their scheduled values. With the integration of higher levels of renewable energy sources such as wind power generation in future power systems, performing frequency regulation using only conventional generators may become technically difficult because of the rapid power imbalances and the insufficient regulation reserve capacity [1-3].

Increasing power system flexibility by responsive demand may become one of the solutions to this problem. Recently, the utilization of demand-side resources such as refrigerators, freezers, heat pump water heaters and air-conditioning loads for providing the needed fast-response regulation services has been shifting into focus [4-7]. One of the reasons why this has not been done is the perceived difficulties in dealing with many small loads rather than a limited number of generating units. In particular, the costs associated with the two-way communications appear to be insurmountable obstacles.

The anticipated electrification of transportation via electric vehicles (EVs) may offer another vast potential of new forms of responsive demand. In China, the cumulative

$\dagger \quad$ Corresponding Author: State Key Laboratory of Advanced Electromagnetic Engineering and Technology (Huazhong University of Science and Technology), Wuhan, China. (liyinhong@hust.edu.cn)

* State Key Laboratory of Advanced Electromagnetic Engineering and Technology (Huazhong University of Science and Technology), Wuhan, China. (pingpx@163.com,dongyuanshi@hust.edu.cn)

Received: August 31, 2014; Accepted: October 21, 2015 sales of battery electric vehicles (BEVs) and plug-in hybrid electric vehicles (PHEVs) are expected to reach five million in 2020 [8]. The large-scale implementation of EVs will provide sufficient battery energy storage for power grid. Integration of EVs into AGC will change the conventional control strategies and improve the performance of power system frequency control.

The existing research about EVs providing frequency regulation is mainly based on the vehicle-to-grid (V2G) [911] framework, which is to manage and control the EVs plugged in the charging outlets. Most of the technical issues are limited to the interaction of the individual vehicle and the power grid in the form of decentralized charging strategies [12] or autonomous V2G control [13]. In [14], an efficient scheduling method for each vehicle regarding the V2G frequency regulation service has been studied. The system frequency fluctuations are evaluated in [15] based on dynamic simulation of applying V2G to AGC system. In [16], the method for dispatching the control signal is presented according to the response speed and controllable capability. Some studies have also addressed the V2G control strategies for plugged EVs supporting primary frequency regulation [17-19]. However, the basic premise of $\mathrm{V} 2 \mathrm{G}$ control is that EVs can connect to grid for a long time in order to ensure a sufficient regulation capacity. If there are not enough EVs, the randomness of user mobility will affect the controllable capacity.

Some countries like Israel, Australia, Denmark and China have presented the business model of battery swapping to recharge EVs. The batteries are charged and managed in the centralized battery swapping stations (BSS). 
The energy storage in BSS will provide a new responsive resource for power system. Published literatures have addressed the benefits of BSS to EV owners and utilities [20-22]. In [23], an optimal recharging strategy for BSS to buy and sell the electricity through the day-ahead market in France has been studied. For considering the interaction between BSS and power grid, the operation cost can be significantly reduced [24, 25]. However, the application of BSS to the system frequency regulation has rarely been studied.

This paper intends to evaluate the effects of BSS energy storage on the dynamic performance of AGC under step and random load disturbance. Section 2 gives a description of the station-to-grid (S2G) concept for the interaction of BSS and power grid. An aggregated S2G model considering the swapping demand of EVs and the available regulation reserve is proposed in section 3 . The contributions of S2G to AGC are verified through a twoarea interconnected system simulation in section 4. Finally, the conclusions are made in section 5 .

\section{Station-to-Grid}

\subsection{The concept of S2G}

At a BSS, the automated swapping platform will replace the depleted battery with a fully-charged one. This process can be realized in less than 5 minutes. There will be more and more BSSs in the future to provide swapping services for the increasing EVs. Integrating the BSS batteries into power systems for providing ancillary services has a great significance in power system operation and control. To achieve this goal, it needs to present a new conceptual framework for the application of BSS energy storage. In this paper, we call it station-to-grid (S2G).

The basic concept of $\mathrm{S} 2 \mathrm{G}$ is that BSS provides services to the grid. BSS can charge during low-demand time and discharge when power is needed by the grid. Its difference with the conventional storage is that BSS needs to meet the swapping demand for EVs.

Fig. 1 schematically illustrates the connections among vehicles, BSS and the electric power grid. The power energy flows from power plant through the grid to electricity users, and flows back to the grid from BSS when it is needed. The EV operation and management center (OMC) is responsible for communicating with the grid operator, managing the BSS batteries and guiding EVs to charge or recharge. The grid control center collects data from EV OMC, power plant and grid, then it sends control signals to BSS through the communication network. The EVs and batteries can be integrated into the internet of things with the wireless communication technologies such as ZigBee, RFID and 3G/GPRS, so that EV OMC can know the vehicle location and battery state, and guide the EVs to the nearest BSS for swapping.

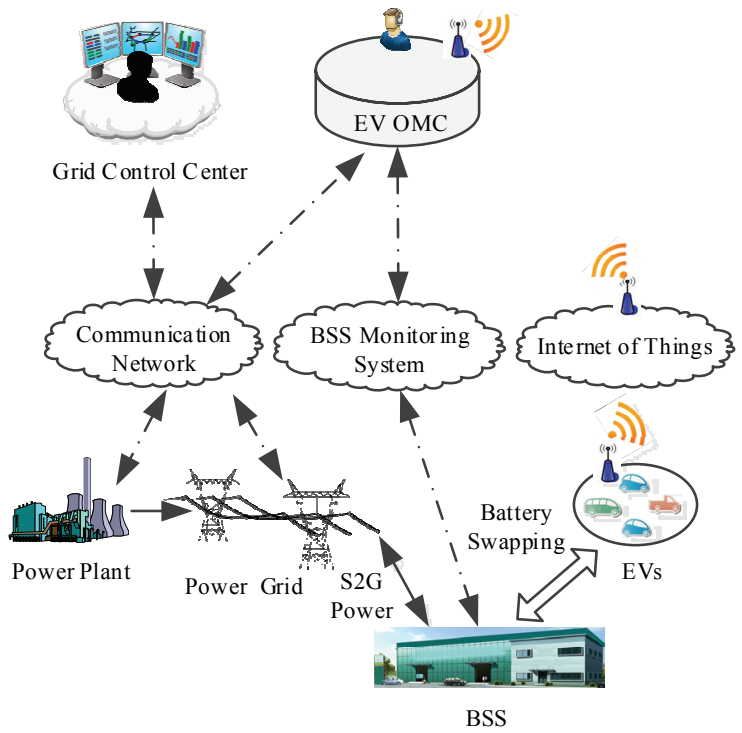

Fig. 1. Illustrative schematic of proposed S2G connections

\subsection{S2G participating in AGC}

The batteries in BSS are very suitable for frequency regulation because of the rapid response capability. When a disturbance occurs in the control area, the AGC will order the BSSs to change their power consumption or output by sending set-points to the EV OMC under its domain. Then, the EV OMC will distribute these set points among the BSSs according to their SOCs at that time. The difference between BSS and conventional battery energy storage (BES) is that the BSS storage is located in the demand side. The S2G power must be limited by the swapping demands.

\section{Frequency Analysis Model with S2G}

\subsection{Available regulation reserves (ARR)}

In order to determine the regulation reserves available from BSS in the electricity market, the OMC needs to forecast the EV swapping demand of the second day ahead. It is usually calculated from the historical data. Unless EVs have been implemented in a large scale, it will be hard to get the actual operating data. This paper uses the queuing theory [26] to determine the available regulation reserves (ARR) for simplicity.

It is assumed that each BSS manage its batteries separately, i.e. not considering the exchange between BSSs. The transition process of battery in BSS can be described as follows:

1) EV arriving for recharge, a depleted battery is replaced with a fully charged one;

2) The depleted batteries wait to be recharged until an arbitrary recharging device becomes available;

3) The depleted batteries start to be recharged as soon as 


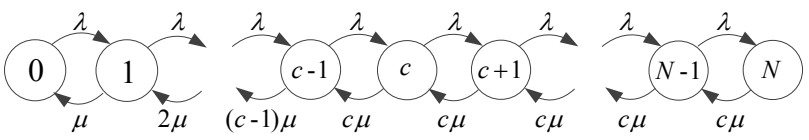

Fig. 2. State transition diagram of BSS

the recharging device is available;

4) After the batteries are fully recharged, they will be transported to the stock zone to set aside for being swapped into next EVs.

It can be seen that there will be three $\dddot{k}$ inds of battery states, i.e., waiting to be charged, charging on the device, and being fully charged. We define that the total number of batteries in a station is $N$, the number of charging device is $c$ (it means that $c$ batteries can be simultaneously charged in the station), and the number of batteries need to be recharged (i.e. sum of the waiting and charging batteries) is $n$. Then, the number of batteries in each phase is described as follows:

$$
\left\{N_{w}, N_{c}, N_{f}\right\}= \begin{cases}\{n-c, c, N-n\} & (c \leq n \leq N) \\ \{0, n, N-n\} & (0 \leq n \leq c)\end{cases}
$$

where $N_{w}$ is the number of batteries waiting to be recharged, $N_{c}$ is the number of batteries being recharged, and $N_{f}$ is the number of batteries that have been fully recharged. Note that $N$ and $c$ are both constants. We can confirm the state of BSS if $n$ is determined. Assumed that EVs arrive into BSS according to a homogeneous Poisson process with the average arrival rate $\lambda$, and charging time follows exponential distribution whose service rate is $\mu$, the state transition diagram of BSS can be depicted as Fig. 2 .

This transition process is a one-dimensional birth and death process. In the steady state, the system equilibrium equation is:

$$
\begin{cases}\lambda p_{0}=\mu p_{1} & (n=0) \\ (\lambda+n \mu) p_{n}=\lambda p_{n-1}+(n+1) \mu p_{n+1} & (1 \leq n<c) \\ (\lambda+c \mu) p_{n}=\lambda p_{n-1}+c \mu p_{n+1} & (c \leq n<N) \\ c \mu p_{N}=\lambda p_{N-1} & (n=N)\end{cases}
$$

It can be seen that EVs at a BSS can be regarded as queuing customers to be served in an $M / M / c / N$ queue, where $M / M$ denote the inter-arrival time of customers and the service time of a customer following the exponential distribution respectively, $c$ denotes the maximum customer number (i.e. the number of swapping devices) being served at the same time, and $N$ denotes the maximum number of batteries. The system state probability can be calculated as:

$$
p_{0}=\left(\sum_{n=0}^{c} \frac{(c \rho)^{n}}{n !}+\frac{c^{c}}{c !} \sum_{n=c+1}^{N} \rho^{n}\right)^{-1}
$$

$$
\begin{aligned}
& p_{n}= \begin{cases}\frac{(c \rho)^{n}}{n !} p_{0} & (n \leq c) \\
\frac{c^{c}}{c !} \rho^{n} p_{0} & (c \leq n \leq N)\end{cases} \\
& \rho=\frac{\lambda}{c \mu}
\end{aligned}
$$

where $p_{i}(i=0,1, \ldots, n)$ is the probability of each state and $\rho$ is the occupation rate per swapping device.

In order to provide a sufficient service level, there should be a safety stock in BSS. We define that the number of batteries that are fully charged and set aside to be swapped into EVs is $N^{*}\left(c<N^{*} \leq N\right)$. When the number of EV waiting for swapping has reached $N^{*}$, the service demand of new EV will be refused. Then, the loss of customer probability $(L O C P)$ due to the service refusal is:

$$
L O C P=\frac{c^{c}}{c !} \rho^{N^{*}}\left(\sum_{k=0}^{c} \frac{(c \rho)^{k}}{k !}+\frac{c^{c}}{c !} \cdot \frac{\rho\left(\rho^{c}-\rho^{N^{*}}\right)}{1-\rho}\right)^{-1}
$$

The safety stock $N^{*}$ can be obtained by reducing $L O C P$ to a certain targeted level $\delta$ (i.e. make $L O C P \leq \delta$ ). For $\mathrm{EV}$ $\mathrm{OMC}$, it can be regarded as the stationary demand of EV swapping because the allowable loss probability has been considered.

Only the batteries connected to the grid can participate in frequency control. It can be assumed that the $N^{*}$ batteries scheduled for EV swapping demand will not provide the regulation reserves (stacked in swapping zone and not connected to grid). The positive reserve $\mathrm{ARR}^{+}$and negative reserve $\mathrm{ARR}^{-}$then can be calculated as:

$$
\begin{aligned}
\mathrm{ARR}^{+} & =\left(N_{t}-N^{*}\right) \cdot C_{b_{-} i n v}-P_{i n i} \\
\mathrm{ARR}^{-} & =-\left(N_{t}-N^{*}\right) \cdot C_{b_{-} i n v}-P_{i n i}
\end{aligned}
$$

where $N_{t}$ is the total number of batteries, $C_{b \text { inv }}$ is the battery inverter capacity, and $P_{i n i}$ is the initial set-point for BSS.

\subsection{Aggregated S2G model}

While in aggregating the BSS to an equivalent resource, the dispatch method of AGC signal must be considered. In this paper, we use the $S O C$ synchronous control method [27] to dispatch the AGC signal from the grid control center, as shown in Fig. 3. It is assumed that there are $i$ batteries in the BSS that can provide regulation service. The grid control center sends AGC signal to BSS every one second. The charge and discharge of the controllable batteries are restricted by their inverter capacity and their $S O C$ limit. Each battery informs the BSS whether it is 


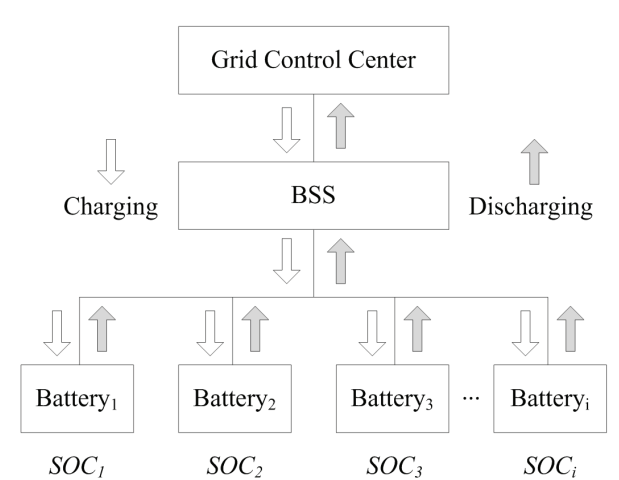

Fig. 3. Dispatching method of AGC signal in BSS

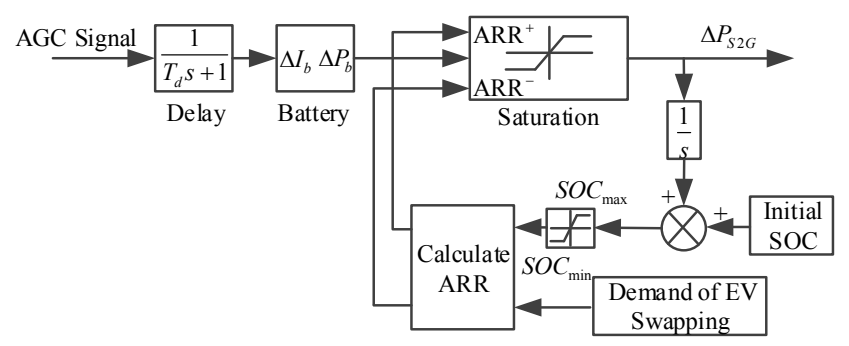

Fig. 4. Aggregated S2G model

controllable or not, and sends its $S O C$ every 30 seconds. After receiving the signal from the grid control center, the BSS dispatches the control signal as follows. The charging and discharging states of batteries are determined according to their SOCs. The charging signal is dispatched to the batteries in the ascending order of $S O C$, while the discharging signal is dispatched to the batteries in the descending order. As a result, the SOCs of the controllable batteries can be synchronized and the BSS can be designed as one large aggregated model.

Fig. 4 shows the aggregated S2G model considering the constraints of ARR. The input of this model is AGC signal for BSS and the output is the total charging/discharging power of BSS, which is controlled based on the ARR at that time. The communication delay is approximated by the first order model with time delay denoted by $T_{d}$. The Thevenin-based battery model [15] which consists of an open circuit voltage source in series with an internal resistance and a parallel RC network is used to compute the output of battery. In view of the real battery protection to avoid damage of the battery and to preserve battery life, the battery power is limited to ARR dynamically according to the allowable $S O C$ range and demand of EV swapping. In particular, if the battery $S O C$ goes out of the range $\left[S O C_{\min }, S O C_{\max }\right]$, ARR will be set to zero to avoid the overcharging or over discharging. That means the battery will not take part in AGC services.

\subsection{Modeling of two-area AGC with S2G}

To investigate the effects of S2G regulation, a two-area

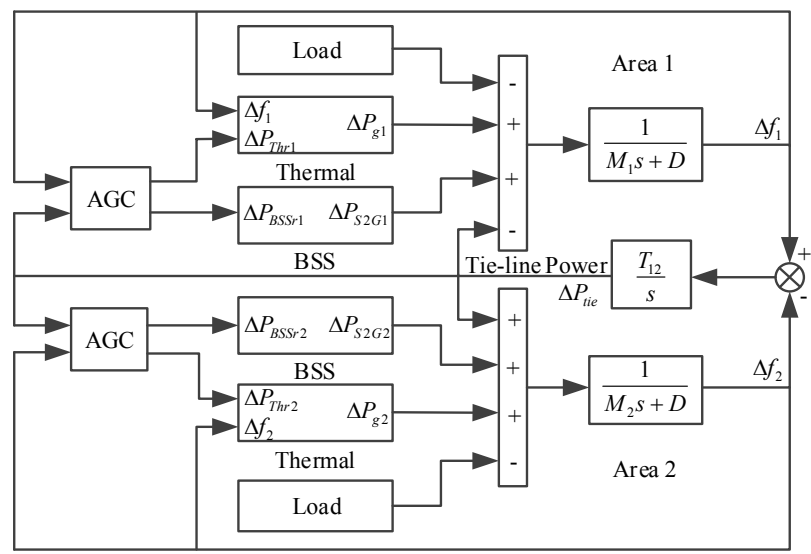

Fig. 5. Two-area interconnected system AGC model

interconnected AGC model is used in this paper. The twoarea interconnected power system linearized around an operating reference point can be described by the standard state space equation as

$$
\dot{\mathrm{X}}=A X+M U+\Gamma P
$$

where $X, U$, and $P$ are the state, control and disturbance vectors, respectively, and $A, M$, and $\Gamma$ are constant matrices of appropriate dimensions which in turn depend on the system parameters and the operating point. For a two-area AGC system with S2G,

$$
\begin{gathered}
X=\left[\Delta f_{1}, \Delta f_{2}, \Delta P_{t i e}, \Delta P_{g 1}, \Delta P_{g 2}, \Delta P_{S 2 G 1}, \Delta P_{S 2 G 2}\right]^{T} \\
U=\left[\Delta P_{T h r 1}, \Delta P_{T h r 2}, \Delta P_{B S S r 1}, \Delta P_{B S S r 2}\right]^{T} \\
P=\left[\Delta P_{L 1}, \Delta P_{L 2}\right]^{T}
\end{gathered}
$$

where $\Delta f_{1}$ and $\Delta f_{2}$ are the frequency deviation of area 1 and area $2, \Delta P_{\text {tie }}$ is the tie-line power deviation, $\Delta P_{g 1}, \Delta P_{g 2}$, $\Delta P_{S 2 G 1}$, and $\Delta P_{S 2 G 2}$ are the outputs of thermal generators and BSS in area 1 and area 2 , respectively, $\Delta P_{T h r 1}, \Delta P_{T h r 2}$, $\Delta P_{B S S r 1}$, and $\Delta P_{B S S r 2}$ are the reference set-points from the AGC control center, and $\Delta P_{L 1}, \Delta P_{L 2}$ are the load disturbance in the two areas, respectively.

The area control error $(A C E)$ of area $i$ which reflects the power imbalance is given by the equation $A C E_{i}=\Delta P_{t i e}+B_{i}$ $\Delta f_{i},(i=1,2)$, where $B_{i}$ is the frequency bias factor. The AGC operation is accomplished by tie-line bias control (TBC) in the two areas to restore the frequency and tie-line power to the scheduled values.

Fig. 5 shows the block diagram of an AGC model integrating the thermal generation units and the aggregated S2G. This model is used to evaluate the dynamic performance of AGC, including an aggregated BSS in each area. The models of thermal generators are those used in [28], which consider the governor dead-band and generation rate constraint. It is assumed that the discharging power of BSS is positive. 


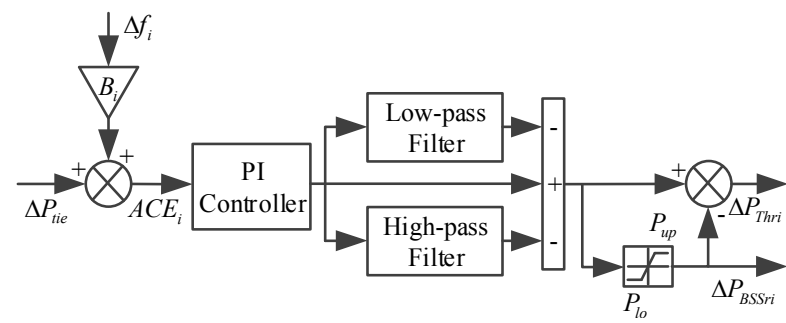

Fig. 6. Coordinated control of thermal units and BSS

\subsection{Coordinated control scheme}

The coordinated control scheme is depicted in Fig.6. The control signal is firstly passed through a low-pass filter and a high-pass filter. The low-pass filter with a time constant of $10 \mathrm{~min}$ is used to filter out the long-cyclic component of the control signal. The high-pass filter with a time constant of $10 \mathrm{~s}$ is used to filter out the fast-cyclic component of the control signal. These two components are not entered to the conventional generators and BSSs. By using this method, only the short-cyclic component whose range is several ten seconds to several minutes is responded by the regulation units. The AGC order of the thermal units are found by subtracting the insufficiency of S2G power.

\section{Simulation Analysis}

It is assumed that there is a BSS in each area and the number of controllable batteries are 1000. The maximum charging/discharging power and average capacity of each battery is $10 \mathrm{~kW}, 40 \mathrm{kWh}$, respectively.

The system base power is $2000 \mathrm{MW}$. It is assumed that EVs go to BSS for battery swapping every two minutes (i.e. $\lambda=0.5$ ) and the average service time is seven minutes (i.e. $\mu=0.14$ ). The number of service window is four (i.e. $c=4$ ) in each BSS. The EV OMC obtains the scheduled reserves for batteries swapping by reducing $L O C P$ below 0.001 (i.e. $\delta=0.001)$. Then ARR can be calculated according to (1)-(7). As a result, $\mathrm{ARR}^{+}$is 0.0018 p.u.MW, and $\mathrm{ARR}^{-}$is -0.0038 p.u.MW. The batteries that are needed to meet the swapping demand have been fully charged and set aside for swapping in advance. Under this assumption, the ARR will keep constant at the simulation time scale.

It is assumed that the initial current is $4.426 \mathrm{kA}$, and the initial SOC of batteries is 0.85 . The other parameters are listed in Table 1. The parameters of area 1 and area 2 are assumed to be identical. The simulation is conducted under the step load disturbance and random load disturbance in order to evaluate the short term and long term frequency control performance.

\subsection{Step load disturbance}

It is assumed that a step increased load of $0.5 \%$ occur at the initial time in area 1 . The simulation results are shown
Table 1. Simulation Parameters

\begin{tabular}{c|c}
\hline Parameters & Values \\
\hline Inertia constant $M_{i}(\mathrm{~s})$ & 0.1667 \\
\hline Load damping $D$ (p.u.MW/Hz) & 0.008333 \\
\hline Synchronized coefficient $T_{12}$ (p.u.MW/radian) & 0.545 \\
\hline Units droop $R_{i}(\mathrm{~Hz} / \mathrm{p} . \mathrm{u} . \mathrm{MW})$ & 2.4 \\
\hline Frequency bias coefficient $B_{i}(\mathrm{p} . \mathrm{u} . \mathrm{MW} / \mathrm{Hz})$ & 0.425 \\
\hline Time constant of governor $T_{g i}(\mathrm{~s})$ & 0.08 \\
\hline Time constant of turbine $T_{t i}(\mathrm{~s})$ & 0.3 \\
\hline Time constant of low-pass filter $T_{L F i}(\mathrm{~s})$ & 600 \\
\hline Time constant of high-pass filter $T_{H F i}(\mathrm{~s})$ & 10 \\
\hline$S O C_{\min }$ & 0.8 \\
\hline$S O C_{\max }$ & 0.9 \\
\hline Initial set-point for BSS. $P_{i n i}(\mathrm{p} . \mathrm{u} . \mathrm{MW})$ & 0.001 \\
\hline BSS control delay $T_{d}(\mathrm{~s})$ & 0.026 \\
\hline Battery internal resistance $R_{0}(\Omega)$ & 0.013 \\
\hline Polarized resistance $R_{p}(\Omega)$ & 0.001 \\
\hline Polarized capacitance $C_{\mathrm{p}}(\mathrm{F})$ & 1 \\
\hline
\end{tabular}

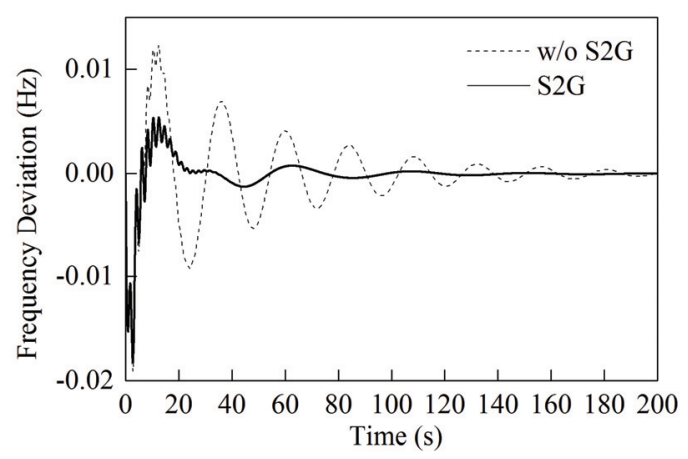

Fig. 7. Frequency deviation of area 1

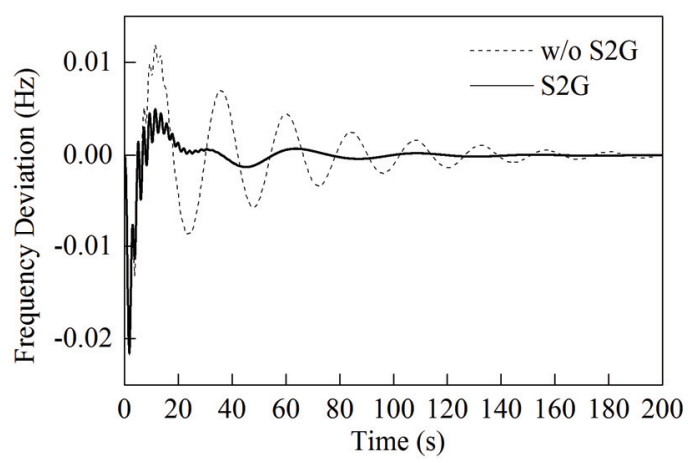

Fig. 8. Frequency deviation of area 2

as Fig. 7-12.

Fig. 7-8 show the frequency deviation fluctuations of area 1 and area 2 with and without S2G, respectively. It can be seen that the frequency fluctuations are suppressed significantly by the $\mathrm{S} 2 \mathrm{G}$ regulation. The peak overshoot of frequency deviation in area 1 is reduced from $0.0123 \mathrm{~Hz}$ to $0.00534 \mathrm{~Hz}$, while from $0.0119 \mathrm{~Hz}$ to $0.00491 \mathrm{~Hz}$ in area 2 . Fig. 9 shows that S2G can also suppress the tie-line power fluctuations and reduce the settling time. Fig. 10 - 12 show the outputs of BSS and thermal units. It can be seen that the outputs of units are reduced due to the $\mathrm{S} 2 \mathrm{G}$ regulation. 


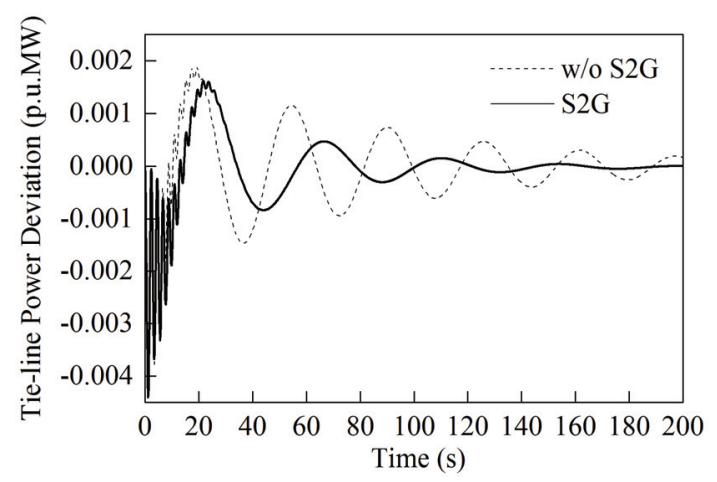

Fig. 9. Tie-line power deviation

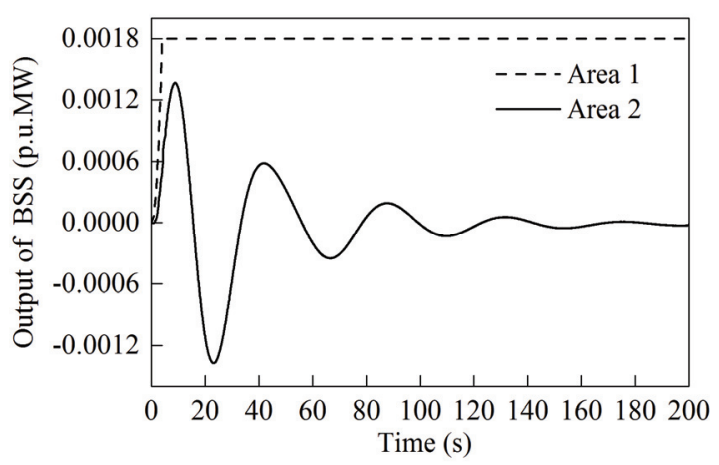

Fig. 10. Output of BSS

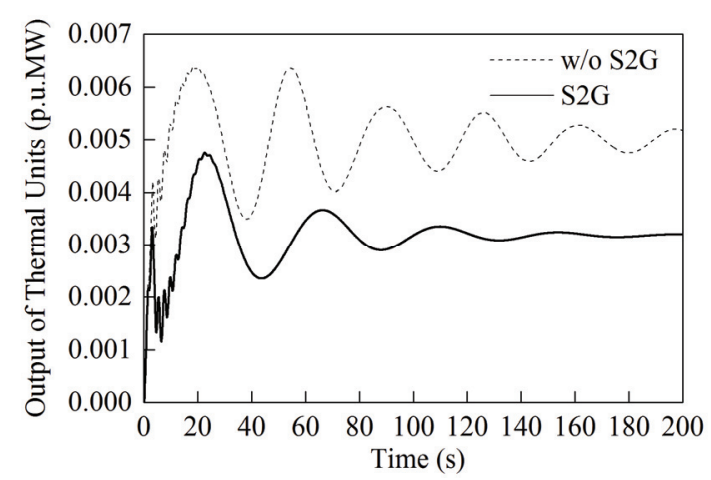

Fig. 11. Output of thermal units in area 1

The output of BSS in area 1 is up to its $A R R^{+}$quickly, while in area 2 , it tends to 0 . In a steady state, the outputs of BSS and units in area 1 are 0.0018 p.u.MW and 0.0032 p.u.MW, respectively, while in area 2 , they are both 0 . That is because the load disturbance in area 1 will be balanced only by its owned regulation resources. Without S2G, the imbalance of power in area 1 is eliminated only by increasing the conventional thermal units. Hence, the output of thermal units in area 1 tends to 0.005 p.u.MW.

\subsection{Random load disturbance}

The random load fluctuation of a real interconnected system can be simulated approximately by a dynamic fluctuation load model [29]. It consists of base and fringe

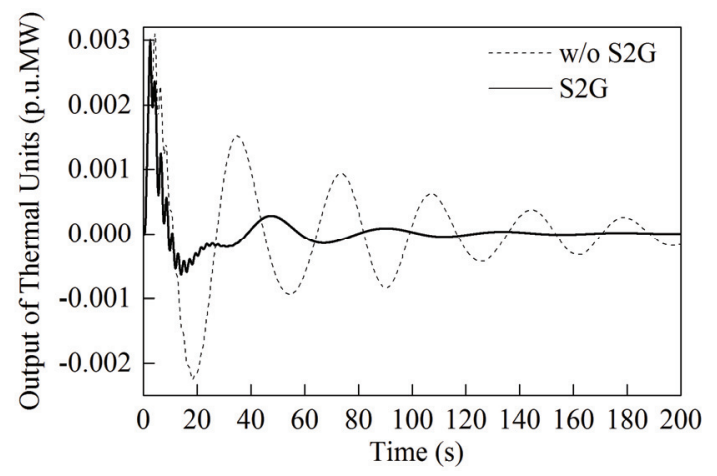

Fig. 12. Output of thermal units in area 2

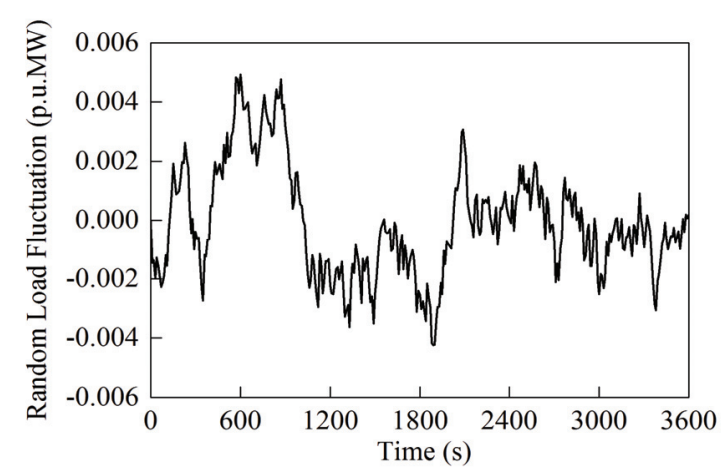

Fig. 13. Random load fluctuation

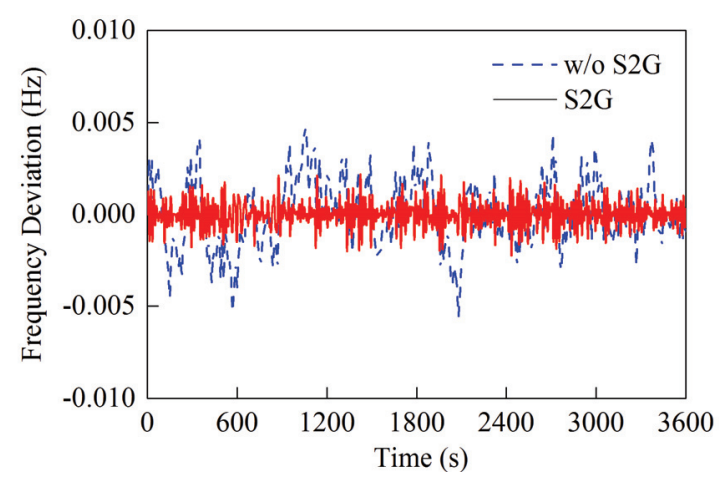

Fig. 14. Frequency deviation of area 1

components obtained from the white noise generators. Fig. 13 shows the random load fluctuation in one hour. The maximum fluctuation band is 0.005 p.u.MW.

It is also assumed that the random load fluctuation occurs in area 1. Fig. 14-15 show the simulation results of frequency deviation in area1 and area 2, respectively. It can be seen that the fluctuations of frequency deviation in both areas has been greatly suppressed. Fig. 16 shows the simulation results of tie-line power deviation. Like the results of frequency deviation, S2G power from BSS can also suppress the fluctuations of tie-line power deviations. Fig. 17-19 show the outputs of BSS and thermal units. It can be seen that the outputs of units are reduced because S2G participating in the AGC program. The output of BSS in area 1 is limited in the range from $\mathrm{ARR}^{-}$to $\mathrm{ARR}^{+}$. 


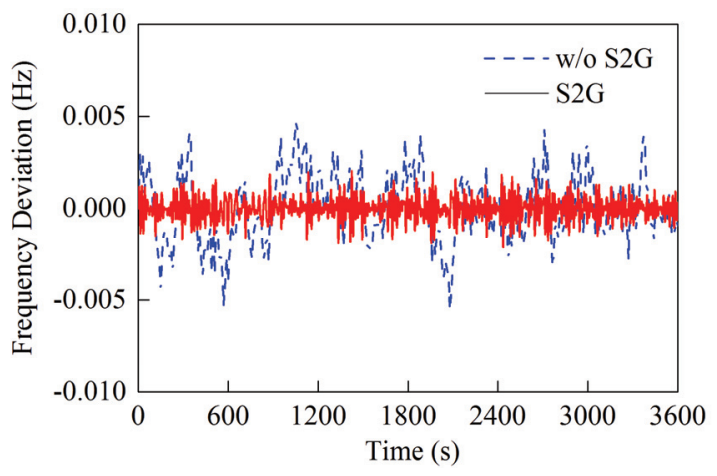

Fig. 15. Frequency deviation of area 2

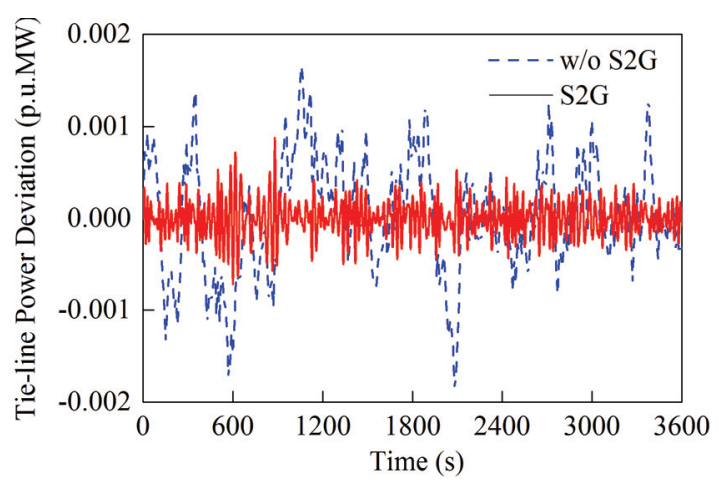

Fig. 16. Tie-line power deviation

Table 2. Quality of frequency deviation

\begin{tabular}{l|l|l|l|l}
\hline & \multicolumn{2}{|l|}{ Area 1 } & \multicolumn{2}{l}{ Area 2 } \\
\cline { 2 - 5 } & w/o S2G & with S2G & w/o S2G & with S2G \\
\hline Max $[\mathrm{Hz}]$ & 0.00465 & 0.00218 & 0.00463 & 0.00203 \\
\hline Min $[\mathrm{Hz}]$ & -0.00555 & -0.00227 & -0.00554 & -0.00213 \\
\hline RMS $[\mathrm{Hz}]$ & 0.00179 & 0.000639 & 0.00178 & 0.00059 \\
\hline
\end{tabular}

Table 3. Quality of tie-line power deviation

\begin{tabular}{l|l|l}
\hline & w/o S2G & with S2G \\
\hline Max [p.u.MW] & 0.00165 & 0.00088 \\
\hline Min [p.u.MW] & -0.00183 & -0.00072 \\
\hline RMS [p.u.MW] & 0.00062 & 0.00019 \\
\hline
\end{tabular}

In order to evaluate the suppression effects of $\mathrm{S} 2 \mathrm{G}$ power quantitatively, some evaluation indexes such as maximum (Max), minimum (Min) and root mean square (RMS) values of the frequency and tie-line power deviations are used. The results are listed in Table 2 and Table 3. It can be seen that the values of these indexes are reduced by $46.67 \%-69.35 \%$ because of the S2G regulation.

\section{Conclusion}

This paper explores how the demand-side BSS energy storage could contribute to AGC. A conceptual framework of S2G is proposed for BSS giving benefits to power grid. The integration of S2G into AGC may change the con-

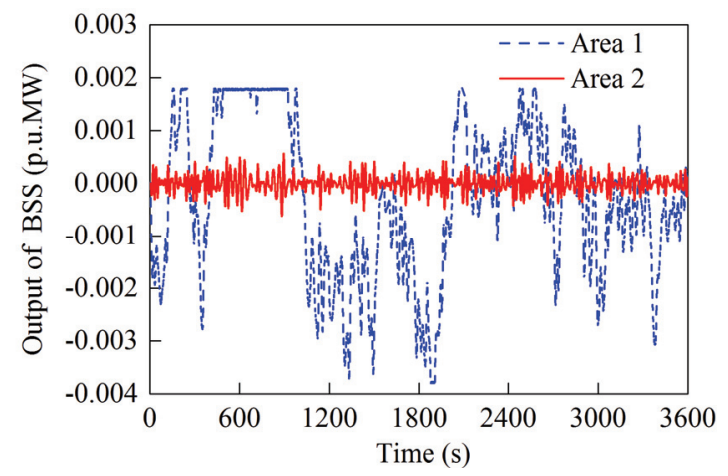

Fig. 17. Output of BSS

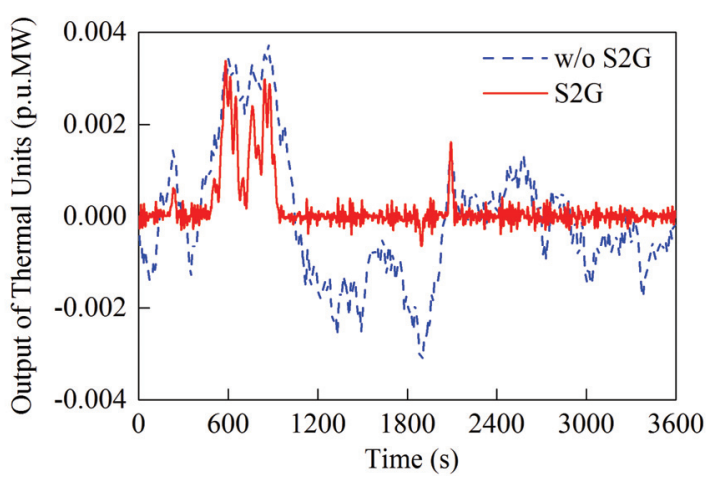

Fig. 18. Output of thermal units in area 1

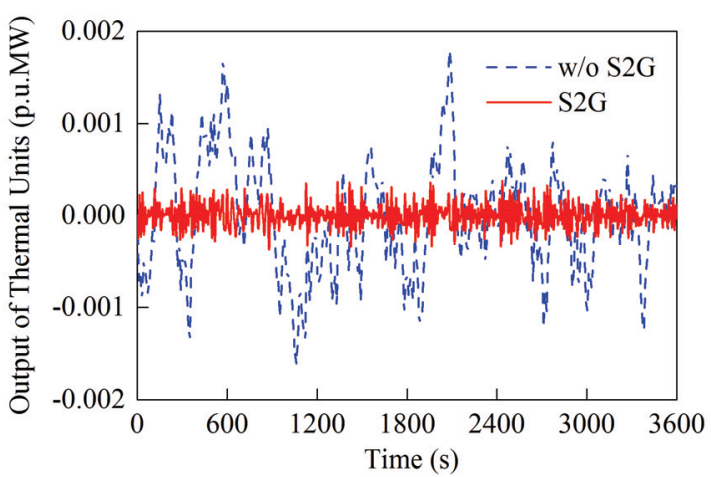

Fig. 19. Output of thermal units in area 2

ventional control strategies that only regulate AGC units, and make the demand-side BSS energy storage participate in frequency regulation. The S2G model and control scheme are proposed to evaluate the effects on AGC. The results show that S2G can significantly suppress the frequency deviation and tie-line power fluctuations and reduce the regulation of AGC units, thus improve the dynamic performance of frequency control.

Although the business model of battery swapping is facing many challenges such as safety requirements, battery standards, and swappable design, it is still an effective measure to shorten the recharging time of EV batteries and provides a large convenience for users extremely in large cities or in densely populated regions. Once the BSS have been implemented in a large scale, they 
will provide new responsive resources in the demand side for supporting power grid services, especially in power system frequency and voltage regulation.

The demand for battery swapping in BSS may fluctuate significantly under a large time window (for example, one day). The dynamic fluctuation of ARR in a day will be considered in our future research.

\section{Acknowledgements}

This work was supported by the National High Technology Research and Development of China (863 Program) (2011AA05A109).

\section{References}

[1] R. Doherty, A. Mullane, G. Nolan, D. Burke, A. Bryson, and M. O'Malley, "An assessment of the impact of wind generation on system frequency control," IEEE Trans. Power Syst., vol. 25, no. 1, pp. 452-460, Feb. 2010.

[2] R. G. de Almeida and J. A. P. Lopes, "Participation of doubly fed induction wind generators in system frequency regulation," IEEE Trans. Power Syst., vol. 22, no. 3, pp. 944-950, Aug. 2007.

[3] Y. Xue and N. Tai, "Review of contribution to frequency control through variable speed wind turbine," Renew. Energy, vol. 36, no. 6, pp. 1671-1677, Jun. 2011.

[4] X. Zhao, J. Ostergaard, and M. Togeby, "Demand as Frequency Controlled Reserve," IEEE Trans. Power Syst., vol. 26, no. 3, pp. 1062-1071, Aug. 2011.

[5] M. D. Ilic, N. Popli, J. Joo, and Y. Hou, “A possible engineering and economic framework for implementing demand side participation in frequency regulation at value" in Proc. IEEE PES Gen. Meet., Detroit, MI, Jul. 2011, pp. 1-7.

[6] J. Kondoh, N. Lu, and D. J. Hammerstrom, "An Evaluation of the Water Heater Load Potential for Providing Regulation Service," IEEE Trans. Power Syst., vol. 26, no. 3, pp. 1309-1316, Aug. 2011.

[7] M. García, F. Bouffard, and D. S. Kirschen, "Decentralized Demand-Side Contribution to Primary Frequency Control," IEEE Trans. Power Syst., vol. 26, no. 1, pp. 411-419, Feb. 2011.

[8] Y. Song, X. Yang, and Z. Lu, "Integration of Plug-in Hybrid and Electric Vehicles: Experience from China," in Proc. IEEE PES Gen. Meet., Minneapolis, MN, Jul. 2010, pp.1-6.

[9] W. Kempton and J. Tomic, "Vehicle-to-grid power fundamentals: Calculating capacity and net revenue," J Power Sources, vol. 144, no. 1, pp. 268-279, Apr. 2005.
[10] J. Tomic and W. Kempton, "Using fleets of electricdrive vehicles for grid support," J. Power Sources, vol. 168, no. 2, pp. 459-468, Jun. 2007.

[11] D. Corey, K. Whitea, and M. Zhang, "Using vehicleto-grid technology for frequency regulation and peak-load reduction," J Power Sources, vol. 196, no. 8, pp. 3972-3980, Apr. 2011.

[12] X. Luo, S. Xia, and K.W. Chan, "A decentralized charging control strategy for plug-in electric vehicles to mitigate wind farm intermittency and enhance frequency regulation," J Power Sources, vol. 248, pp. 604-614, 2014

[13] Y. Ota, H. Taniguchi, T. Nakajima, K. M. Liyanage, J. Baba, and A. Yokoyama, "Autonomous Distributed V2G (Vehicle-to-Grid) Satisfying Scheduled Charging," IEEE Trans. Smart Grid, vol. 3, no. 1, pp. 559564, Mar. 2012.

[14] S. Han, S. H. Han, and K. Sezaki, "Development of an optimal vehicle-to-Grid aggregator for frequency regulation," IEEE Trans. Smart Grid, vol. 1, no. 1, pp. 65-72, Jun. 2010.

[15] J. R. Pillai and B. B. Jensen, "Integration of vehicleto-grid in the Western Danish power system," IEEE Trans. Sustainable Energy, vol. 2, no. 1, pp. 12-19, Jan. 2011.

[16] T. Masuta and A. Yokoyama, "Supplementary Load Frequency Control by Use of a Number of Both Electric Vehicles and Heat Pump Water Heaters," IEEE Trans. Smart Grid, vol. 3, no. 3, pp. 1253-1262, Sept. 2012.

[17] H. Yang, C. Y. Chung, and J. Zhao, "Application of Plug-In Electric Vehicles to Frequency Regulation Based on Distributed Signal Acquisition Via Limited Communication," IEEE Trans. Power Syst., vol. 28, no. 2, pp.1017-1026, May. 2013.

[18] Y. Mu, J. Wu, J. Ekanayake, N. Jenkins, and H. Jia, "Primary Frequency Response From Electric Vehicles in the Great Britain Power System," IEEE Trans. Smart Grid, vol. 4, no. 2, pp. 1142-1150, Jun. 2013.

[19] H. Liu, Z. Hu, Y. Song, and J. Lin, "Decentralized Vehicle-to-Grid Control for Primary Frequency Regulation Considering Charging Demands," IEEE Trans. Power Syst., vol. 28, no. 3, pp. 3480-3489, Aug. 2013.

[20] P. Lombardi, M. Heuer, and Z. Styczynski, "Battery switch station as storage system in an autonomous power system: Optimization issue," in Proc. IEEE PES Gen. Meet., Minneapolis, MN, Jul. 2010, pp.1-6.

[21] M. Takagi, Y. Iwafune, K. Yamaji, H. Yamamoto, K. Okano, R. Hiwatari, and T. Ikeya, "Economic Value of PV Energy Storage Using Batteries of Batteryswitch Stations," IEEE Trans. Sustainable Energy, vol. 4, no. 1, pp. 164-73, Jan. 2013.

[22] Y. Miao, Q. Jiang, and Y. Cao, "Battery switch station modeling and its economic evaluation in microgrid," in Proc. IEEE PES Gen. Meet., San Diego, CA, Jul. 
2012, pp. 1-7.

[23] M. Armstrong, C. E. H. Moussa, J. Adnot, A. Galli, and P. Riviere, "Optimal recharging strategy for battery-switch stations for electric vehicles in France," Energy Policy, vol. 60, pp. 569-582, Sept. 2013.

[24] Y. Gao, K. Zhao, and C. Wang, "Economic dispatch containing wind power and electric vehicle battery swap station," in Proc. IEEE PES Transmission and Distribution Conference and Exposition, Orlando, FL, May. 2012, pp. 1-7.

[25] S. Zhang, Z. Hu, Y. Song, H. Liu, and M. Bazargan, "Research on unit commitment considering interacttion between battery swapping station and power grid," Proceedings of the CSEE, vol. 32, no. 10, pp. 49-55, Apr. 2012.

[26] U. N. Bhat, An Introduction to Queueing Theory: Modeling and Analysis in Applications. New York: Birkhäuser Boston \& Springer Science, 2008, pp. 4350.

[27] K. Shimizu, T. Masuta, Y. Ota, and A. Yokoyama, "Load frequency control in power system using vehicle-to-grid system considering the customer convenience of electric vehicle," in Proc. Int. Conf. Power Syst. Technol., Hangzhou, China, Oct. 2010, pp. 1-8.

[28] C. F. Lu, C. C. Liu, and C. J. Wu, "Effect of battery energy storage system on load frequency control considering governor deadband and generation rate constraint," IEEE Trans. Energy Convers, vol. 10, no. 3, pp. 555-561, Sep. 1995.

[29] T. Michigami and T. Ishii, "Construction of fluctuation load model and dynamic simulation with LFC control of DC power system and frequency converter interconnection," in Proc. IEEE Power Eng. Soc. Trans. Distrib. Conf., Yokahama, Japan, Oct. 2002, pp. 382-387.

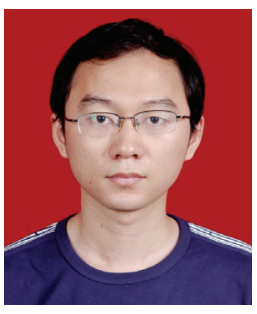

Pingping Xie He received his B.S. degree in Department of Electrical Engineering in Chongqing University in 2009. He is currently working towards the Ph.D. in Huazhong University of Science and Technology in China. His research interests include power system frequency control, vehicle-to-grid, and demand response.

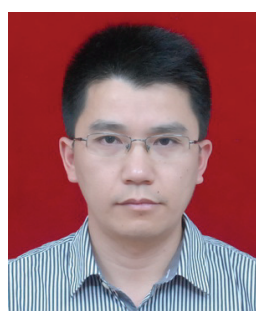

Dongyuan Shi He received the B.S. and $\mathrm{Ph} . \mathrm{D}$. degrees from Huazhong University of Science and Technology (HUST), Wuhan, China, in 1996 and 2002 , respectively. He is currently an Associate Professor in the School of Electrical and Electronic Engineering, HUST. His research interests include power system analysis and application of artificial intelligence in power systems.

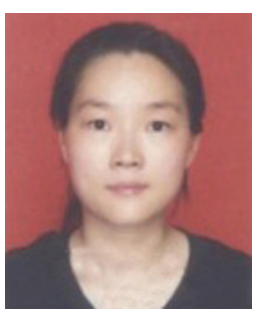

Yinhong Li She received the B.S. and $\mathrm{Ph} . \mathrm{D}$. degrees in power systems from Huazhong University of Science and Technology (HUST), Wuhan, China, in 1998 and 2004, respectively. She is currently an Associate Professor in the School of Electrical and Electronic Engineering, HUST. Her research interests include power system protection, load modeling, and voltage stability analysis. 